\title{
Caso probable de fiebre manchada (Rickettsia felis) transmitida por pulgas
}

\author{
Álvaro A. Faccini-Martínez¹, Elkin G. Forero-Becerra², Jesús A. Cortés-Vecino², Luis J. Polo-Teran², \\ Jorge H. Jácome ${ }^{3}$, Jimmy J. Vargas ${ }^{4}$, Gustavo Valbuena ${ }^{5}$, Marylin Hidalgo ${ }^{1}$ \\ 1 Grupo de Enfermedades Infecciosas, Departamento de Microbiología, Facultad de Ciencias, Pontificia \\ Universidad Javeriana, Bogotá, D.C., Colombia \\ ${ }^{2}$ Laboratorio de Parasitología Veterinaria, Facultad de Medicina Veterinaria y de Zootecnia, Universidad Nacional \\ de Colombia, Bogotá, D.C., Colombia \\ 3 Departamento de Biología, Facultad de Ciencias, Pontificia Universidad Javeriana, Bogotá, D.C., Colombia \\ ${ }^{4}$ Instituto de Genética, Universidad Nacional de Colombia, Bogotá, D.C., Colombia \\ 5 Pathology Department, University of Texas Medical Branch, Galveston, Texas, USA
}

Rickettsia felis es el agente etiológico de la fiebre manchada transmitida por pulgas, cuyo principal vector y reservorio es Ctenocephalides felis. Típicamente, la enfermedad se presenta como fiebre aguda asociada a cefalea, astenia, exantema máculo-papular generalizado y, en algunos casos, con escara de inoculación. En los últimos años, $R$. felis ha venido adquiriendo un papel importante en la etiología del síndrome febril agudo, calificándola como una enfermedad emergente y subdiagnosticada. La inmunofluorescencia indirecta es actualmente el método diagnóstico de referencia. Sin embargo, esta técnica presenta limitaciones relacionadas con la reacción cruzada que existe entre las diferentes especies del género Rickettsia. En el presente reporte se describe el caso de un paciente de 16 años con síndrome febril agudo secundario a infección probable por $R$. felis.

Palabras clave: estudios de casos, Rickettsia felis, fiebre, infecciones por Rickettsia, Colombia. doi: http://dx.doi.org/10.7705/biomedica.v33i0.723

\section{Probable case of flea-borne spotted fever (Rickettsia felis)}

Rickettsia felis is the etiologic agent of flea-borne spotted fever, with Ctenocephalides felis as its main vector and reservoir. Typically, the disease presents as acute fever associated with headache, asthenia, generalized maculo-papular rash, and in some cases, an inoculation eschar. In recent years, $R$. felis has acquired an important role in the etiology of the acute febrile syndrome; it is indeed an emerging infectious disease, albeit underdiagnosed. Indirect immunofluorescence assay (IFA) is currently the reference diagnostic method. However, this technique has limitations related to the cross reactivity among different species of rickettsiae. Herein, we describe a case of a 16 year-old patient with an acute febrile syndrome secondary to probable infection with $R$. felis.

Key words: Case studies, Rickettsia felis, fever, Rickettsia infections, Colombia. doi: http://dx.doi.org/10.7705/biomedica.v33i0.723

Las rickettsiosis son entidades clínicas causadas por bacterias intracelulares del género Rickettsia que afectan al hombre al ser transmitidas por artrópodos vectores (1). Los miembros de este género bacteriano se clasifican actualmente en

Correspondencia:

Marylin Hidalgo, Departamento de Microbiología, Facultad de Ciencias, Pontificia Universidad Javeriana, Carrera $7 \mathrm{~N}^{\circ}$ 43-82, Bogotá, D.C., Colombia

Teléfono: (571) 320 8320, extensión 4153

hidalgo.m@javeriana.edu.co

Recibido: 30/04/12; aceptado:04/10/12 cuatro grupos según sus características genéticas: grupo de las fiebres manchadas ( $R$. rickettsii, $R$. conorii y $R$. parkeri, entre otras), grupo del tifus $(R$. prowazekii y $R$. typhi), grupo transicional $(R$. felis, $R$. akari y $R$. australis) y grupo ancestral $(R$. bellii y $R$. canadensis); este último no tiene un caracter patógeno conocido (1).

Rickettsia felis es el agente etiológico de la fiebre manchada transmitida por pulgas. Ctenocephalides felis es el principal vector y reservorio. Tanto animales domésticos (por ejemplo, gatos y perros)

\section{Contribución de los autores:}

Álvaro Adolfo Faccini se encargó de la revisión de la literatura científica y la redacción del manuscrito.

Marylin Hidalgo asesoró la revisión, sugirió modificaciones de estilo, corrigió el manuscrito y suministró ideas para la discusión

Elkin G. Forero, Jesús A. Cortés, Luis J. Polo, Jorge H. Jácome, Jimmy J. Vargas y Gustavo Valbuena, asesoraron la revisión y son coinvestigadores del proyecto del cual es producto este manuscrito. 
como peridomésticos (por ejemplo, zarigüeyas) son los huéspedes principales utilizados por este artrópodo para desarrollar su ciclo biológico (2).

La primera evidencia de infección humana por $R$. felis data de principios de los años noventa en los Estados Unidos (3); fue descrita como una nueva especie capaz de producir un cuadro clínico similar al del tifus murino (sic.) (R. typhi) consistente en fiebre aguda asociada a cefalea, mialgias, astenia y exantema máculo-papular (4).

La fiebre manchada transmitida por pulgas ha sido descritadesdeentoncesenmuchospaíses, incluyendo Egipto, Francia, Alemania, Corea del Sur, España, Taiwán, Tailandia y los de Norteamérica, entre otros (2,5-8). En Latinoamérica y el Caribe, actualmente se destaca como la especie reportada en el mayor número de países (9). Es evidente, entonces, que $R$. felis está emergiendo y es importante en la etiología del síndrome febril agudo (10-12).

A continuación se presenta la descripción clínica de un adolescente con infección probable por $R$. felis (fiebre manchada transmitida por pulgas).

\section{Presentación del caso}

Se trata de un adolescente de 16 años de edad, procedente del área urbana del municipio de Útica (Cundinamarca), estudiante de secundaria, de raza mestiza, que consultó por un cuadro clínico de tres días de evolución consistente en fiebre subjetiva asociada a astenia, adinamia, mialgias, odinofagia, tos seca y sensación de desvanecimiento en las últimas dos horas. La revisión por sistemas fue negativa. El paciente recibió tratamiento con acetaminofén y amoxicilina, sin presentar mejoría. Los antecedentes clínicos no fueron relevantes. El paciente refirió presencia de caballos, gatos y perros en la zona de potrero ubicada en el peridomicilio. Negó picadura por artrópodos o viajes a otras regiones en el último mes.

Al ingreso hospitalario, el paciente se encontraba en aceptables condiciones generales, hidratado, con una temperatura de $37^{\circ} \mathrm{C}$, mucosas húmedas congestivas y cuello móvil sin adenopatías. No se evidenció exantema en piel ni déficit neurológico. El resto del examen físico era normal. Se dejó en observación con diagnóstico de laringitis viral aguda y se inició tratamiento con acetaminofén y líquidos intravenosos.

En los exámenes iniciales de laboratorio, se encontró: 4,5 leucocitos por 10\% (neutrófilos, $65,2 \%$; linfocitos, $30,2 \%$ ); plaquetas, $173.000 /$ $\mathrm{mm}^{3}$; hemoglobina, $16,8 \mathrm{~g} / \mathrm{dl}$, y hematocrito, $51,5 \%$. Después de 12 horas del ingreso, en el cuadro hemático se encontró leucopenia (2,8 leucocitos por $10 \%$ L; neutrófilos, $48,3 \%$; y linfocitos, $44,3 \%$ ) y trombocitopenia (plaquetas, $145.000 / \mathrm{mm}^{3}$ ).

Para este momento, el paciente continuaba en aceptables condiciones generales sin cambios en el examen físico, hidratado y afebril. Debido al cuadro clínico de ingreso, la zona de procedencia y la leucopenia asociada a trombocitopenia evidenciada en el cuadro hemático de control, se determinó una sospecha clínica de dengue sin signos de alarma. Se hospitalizó y se continuó igual manejo con líquidos intravenosos, antipiréticos y cuadro hemático de control cada 12 horas.

Entre los diagnósticos diferenciales se consideró la infección por rickettsias, dado su papel como causa de síndrome febril agudo en la zona geográfica.

En el quinto día de hospitalización, se tomó una muestra de sangre con consentimiento del paciente para separación de suero y análisis diagnóstico de dengue (IgM ELISA de captura-Panbio Diagnostics ${ }^{\circledR}$ ) y de rickettsiosis (inmunofluorescencia indirecta para la identificación de anticuerpos lgG contra $R$. rickettsii, cepa Taiaçu, $R$. felis y $R$. amblyommii, cepas donada por Marcelo Labruna; $R$. akari, $R$. parkeri y $R$. typhi, cepas donadas por la University of Texas Medical Branch, considerándose una dilución de 1:64 o mayor como positivo).

El paciente permaneció hospitalizado durante seis días hasta evidenciarse resolución tanto de la leucopenia como de la trombocitopenia (cuadro 1). Durante el mismo periodo, permaneció en aceptable estado general salvo por la presencia de náuseas sin vómito en los dos últimos días de hospitalización por lo cual requirió metoclopramida. Tampoco se documentaron nuevos picos febriles, cambios en el examen físico, ni manifestaciones hemorrágicas. Se decidió, entonces, dar al paciente de alta, con cita de control por consulta externa después de 15 días.

Durante la cita de control (15 días después del egreso) se tomó una nueva muestra de suero para procesarlo como fase de convalecencia de rickettsiosis. Se observaron los siguientes resultados: IgM para dengue negativa y elevación significativa de títulos contra $R$. felis (diferencia de cuatro o más títulos entre la muestra de fase aguda y la de convalecencia) (cuadro 2). Los resultados sugirieron el diagnóstico de fiebre manchada transmitida por pulgas. 
Cuadro 1. Evolución de leucocitos y plaquetas durante el tiempo de hospitalización

\begin{tabular}{lcc}
\hline & Leucocitos (por 109/) & Plaquetas $\left(\right.$ por $\left.\mathbf{~ m m}^{3}\right)$ \\
\hline Día de ingreso & 4,5 & 173.000 \\
Día 1 & 2,8 & 145.000 \\
& 2,4 & 146.000 \\
Día 2 & 3,0 & 138.000 \\
& 3,7 & 153.000 \\
Día 3 & 3,4 & 151.000 \\
& 4,1 & 138.000 \\
Día 4 & 4,0 & 159.000 \\
& 4,8 & 159.000 \\
Día 5 & 4,0 & 169.000 \\
Alta & 4,8 & 185.000 \\
& & \\
\hline
\end{tabular}

\section{Discusión}

Desde sus primeras descripciones, la fiebre manchada transmitida por pulgas (también conocida como el tifus de la pulga del gato) (4) se ha considerado como una enfermedad muy similar al tifus murino (sic.) por su presentación clínica y la asociación con los mismos vectores. Actualmente, gracias a la descripción cada vez más frecuente de esta enfermedad en varios países del mundo, es calificada como una rickettsiosis emergente (5). Típicamente, se caracteriza por producir un cuadro febril agudo, asociado a cefalea, astenia, exantema máculo-papular generalizado y, en algunos casos, una lesión costrosa con halo eritematoso circundante, conocida como escara de inoculación (4). Sin embargo, en los estudios llevados a cabo en Kenia y Senegal en los últimos dos años (1012), se describen casos confirmados de infección por $R$. felis, en los cuales la principal manifestación clínica fue la fiebre no asociada a exantema ni a escara de inoculación y, por el contrario, sí a síntomas como los de nuestro caso, que incluyen tos, náuseas, mialgias y odinofagia. Por otro lado, a pesar de no administrar un antibiótico específico contra rickettsias, se evidenció una evolución clínica satisfactoria, sin picos febriles durante toda la hospitalización ni manifestaciones hemorrágicas; esta situación es indicativa del típico curso benigno de esta enfermedad (13).

Dados los hallazgos de laboratorio predominantes en este paciente (leucopenia y trombocitopenia) asociados a un cuadro febril de tres días de evolución y procedencia de área endémica (14), es claro que la primera aproximación diagnóstica debe ser una infección por el virus del dengue, tal cual como se hizo en este caso. Sin embargo, si se observan con detenimiento los cambios en los leucocitos y las plaquetas a lo largo del tiempo, no se apreció una disminución progresiva de estas dos líneas celulares que luego aumentaron de la misma forma, ni una trombocitopenia tan acentuada (característica del dengue) (15); en cambio, se evidenciaron fluctuaciones de la misma bicitopenia, hallazgo también descrito en las infecciones por R. felis (4).

Es de resaltar el antecedente epidemiológico de presencia de animales domésticos en el peridomicilio como un factor de riesgo debido al posible contacto con sus ectoparásitos, ya que $R$. felis no solo se ha encontrado infectando pulgas de la especie $C$. felis, sino también de $C$. canis y otros géneros de pulgas (Pulex, Echidnophaga, Tunga y Xenopsylla, entre otros), incluso, garrapatas y ácaros $(2,16)$.

Respecto al diagnóstico de las rickettsiosis, la inmunofluorescencia indirecta (IFI) (muestras pareadas, por lo menos, con 15 días de diferencia entre una y otra), es actualmente el método de referencia. No obstante, esta prueba presenta limitaciones en cuanto a la reacción cruzada que existe entre las diferentes especies de Rickettsia, siendo necesario utilizar métodos más precisos como la reacción en cadena de la polimerasa (PCR) y el Western blot, este último asociado a la

Cuadro 2. Resultado de IgG contra especies de Rickettsia por inmunofluoresconcia indirecta (IFI) en fase aguda y en fase de convalecencia (15 días de diferencia)

\begin{tabular}{lccl}
\hline Especie de Rickettsia & $\begin{array}{c}\text { IgG fase } \\
\text { aguda (IFI) }\end{array}$ & $\begin{array}{c}\text { IgG fase de } \\
\text { convalecencia (IFI) }\end{array}$ & Interpretación \\
\hline R. rickettsii & $1: 64$ & $1: 64$ & Exposición previa al grupo de las fiebres manchadas \\
R. felis & $<1: 64$ & $1: 256$ & Aumento significativo de títulos \\
R. amblyommii & $<1: 64$ & $<1: 64$ & Dilución negativa para esta especie \\
R. akari & $<1: 64$ & $<1: 64$ & Dilución negativa para esta especie \\
R. parkeri & $1: 64$ & $<1: 64$ & Exposición previa al grupo de las fiebres manchadas \\
R. typhi & $1: 64$ & $1: 128$ & Reacción cruzada con el grupo del tifus \\
\hline
\end{tabular}

* Dilución $\geq 1: 64$ (positivo) 
prueba de absorción cruzada, para la adecuada caracterización de especies (13).

Tratando de prever estos inconvenientes, se procesó el suero (muestra de fase aguda y de convalecencia) frente a diferentes especies de Rickettsia ( $R$. rickettsii, $R$. felis, $R$. amblyommii, $R$. akari, $R$. parkeri y $R$. typhi); a pesar de observarse reacción cruzada (excepto con $R$. amblyommii y $R$. akari), fue claro el aumento y la diferencia significativa de títulos (de cuatro o más títulos entre las muestras de ambas fases) (17) contra $R$. felis (menos de 1:64 y 1:256 en la fase aguda y la de convalecencia, respectivamente). Este hallazgo es importante, ya que se tienen en cuenta para descartarlo, antígenos de especies de Rickettsia pertenecientes al grupo de las fiebres manchadas que podrían estar circulando en el departamento de Cundinamarca, dadas las altas tasas de seroprevalencia previamente reportadas en este departamento $(18,19)$.

Por otra parte, es evidente que la presentación y la evolución clínica de este paciente fueron benignas (disminución de la fiebre sin necesidad de manejo antibiótico, ausencia de gravedad o complicaciones) (13), contrario a los cuadros febriles abruptos con necesidad de manejo antibiótico oportuno, manifestaciones exantemáticas y frecuentes complicaciones de tipo neurológico o pulmonar, característicos de la infección por $R$. rickettsii, especie que ya ha sido aislada en casos mortales de Villeta y Tobia, Cundinamarca (20).

Lo anterior, asociado a un resultado negativo para infección por virus del dengue (IgM), apoya el diagnóstico probable de fiebre manchada transmitida por pulgas.

Finalmente, al ser $R$. felis la especie reportada en el mayor número de países de Latinoamérica y el Caribe (9), incluso ya caracterizada en el departamento de Caldas (Colombia) (21), se hace oportuno tenerla en cuenta como agente etiológico de síndrome febril agudo de naturaleza zoonótica, sin dejar a un lado otros agentes del orden de los rickettsiales (Anaplasmay Ehrlichia), especialmente en el departamento de Cundinamarca, región donde, según los estudios de seroprevalencia, es evidente la circulación del género Rickettsia $(18,19)$.

\section{Agradecimientos}

Al cuerpo médico, al Laboratorio de Bacteriología y al Departamento de Estadística del Hospital Salazar de Villeta, Cundinamarca.

\section{Conflictos de interés}

Durante la realización de este artículo, ninguno de los autores tuvo vinculación con ninguna actividad que pudiera representar conflictos de interés.

\section{Financiación}

La publicación del presente manuscrito es producto del estudio: "Caracterización de factores climáticos y ecológicos de una especie de garrapata y su relación con la epidemiología de la rickettsiosis en un área endémica", patrocinado por Colciencias, código 120351929098.

\section{Referencias}

1. Mansueto P, Vitale G, Cascio A, Seidita A, Pepe I, Carroccio A, et al. New insight into immunity and immunopathology of rickettsial diseases. Clin Dev Immunol. 2012;2-26. http://dx.doi.org/10.1155/2012/967852

2. Reif K, Macaluso K. Ecology of Rickettsia felis: A review. J Med Entomol. 2009;46:723-36.

3. Schriefer M, Sacci J, Dumler J, Bullen M, Azad A. Identification of a novel rickettsial infection in a patient diagnosed with murine typhus. J Clin Microbiol. 1994; 32 : 949-54.

4. Raoult D, Parola P. Rickettsial diseases. New York: Informa Healthcare; 2007. p. 87-96.

5. Pérez-Osorio C, Zavala-Velázquez J, Árias J, ZavalaCastro J. Rickettsia felis as emergent global threat for humans. Emerg infect Dis. 2008;14:1019-23. http://dx.doi. org/10.3201/eid1407.071656

6. Rolain J, Franc M, Davoust B, Raoult D. Molecular detection of Bartonella quintana, B. koehlerae, B. henselae, B. clarridgeiae, Rickettsia felis, and Wolbachia pipientis in cat fleas, France. Emerg Infect Dis. 2003;9:338-42. http:// dx.doi.org/10.3201/eid0903.020278

7. Bernabeu-Wittel $\mathbf{M}$, del Toro $\mathbf{M}$, Noguera $\mathbf{M}$, Muniain M, Cardeñosa N, Márquez F, et al. Seroepidemiological study of Rickettsia felis, Rickettsia typhi, and Rickettsia conorii infection among the population of souther Spain. Eur $\mathrm{J}$ Clin Microbiol Infect Dis. 2006;25:375-81. http://dx.doi. org/10.1007/s10096-006-0147-6

8. Parola P, Sanogo O, Lerdthusnee K. Identification of Rickettsia spp. and Bartonella spp. from the Thai-Myanmar border. Ann N Y Acad Sci. 2003;990:173-81. http://dx.doi. org/10.1111/j.1749-6632.2003.tb07359.x

9. Labruna M, Mattar S, Nava S, Bermúdez S, Venzal J, Dolz Gaby, et al. Rickettsiosis in Latin America, Caribbean, Spain and Portugal. Revista MVZ Córdoba. 2011;16:2435-57.

10. Socolovschi C, Mediannikov O, Sokhna C, Tall A, Diatta G, Bassene H, et al. Rickettsia felis-associated uneruptive fever, Senegal. Emerg Infect Dis. 2010;16:1140-2. http:// dx.doi.org/10.3201/eid1607.100070

11. Richards A, Jiang J, Omulo S, Dare R, Khalif A, Ali A, et al. Human Infection with Rickettsia felis, Kenya. Emerg Infect Dis.2010;16:1081-6.http://dx.doi.org/10.3201/ eid 1607. 091885 
12. Maina A, Knobel D, Jiang J, Halliday J, Feikin D, Cleaveland S, et al. Rickettsia felis Infection in febrile patients, Western Kenya, 2007-2010. Emerg Infect Dis. 2012;18:328-31. http://dx.doi.org/10.3201/eid1802.111372

13. Renvoisé A, Raoult D. An update on rickettsiosis. Med Mal Infect.2009;39:71-81.http://dx.doi.org/10.1016/j.medmal. 2008.11.003,

14. Ministerio de la Protección Social, Instituto Nacional de Salud, Organización Panamericana de la Salud. Guía de atención clínica integral del paciente con dengue. Bogotá, D.C.: Ministerio de la Protección Social, Instituto Nacional de Salud, OPS/OMS; 2010. p. 7.

15 Wilder-Smith A, SchwartzE. Dengue in travelers. N Engl J Med. 2005;353:924-32. http://dx.doi.org/10.1056/NEJMra041927

16. Eisen R, Gage K. Transmission of flea-borne zoonotic agents. Annu Rev Entomol. 2012;57:61-82. http://dx.doi. org/10.1146/annurev-ento-120710-100717

17. Chapman AS, Bakken JS, Folk SM, Paddock CD, Bloch $\mathrm{KC}$, Krusell A, et al. Diagnosis and management of tickborne rickettsial diseases: Rocky Mountain spotted fever, ehrlichioses, and anaplasmosis -United States. A practical guide for physicians and other health-care and public health professionals. MMWR Recomm Rep. 2006;55:1-27.

18. Hidalgo M. Panorama de las enfermedades ocasionadas por rickettsias en Colombia. Biomédica. 2011;31:11-73.

19. Hidalgo M, Sánchez R, Orejuela L, Hernández J, Walker D, Valbuena G. Prevalence of antibodies against spotted fever group Rickettsiae in a rural Area of Colombia. Am J Trop Med Hyg. 2007;77:378-80.

20. Hidalgo $M$, Orejuela L, Fuya $P$, Carrillo $P$, Hernández J, Parra E, et al. Rocky Mountain spotted fever, Colombia. Emerg Infect Dis. 2007;13:1058-60. http://dx.doi.org/10.3201/ eid1307.060537

21. Ramírez-Hernández A, Montoya V, Martínez A, Pérez JE, Mercado M, De la Ossa A, et al. Molecular detection of Rickettsia felis in different flea species from Caldas, Colombia. Am J Trop Med Hyg. 2013;89;453-9. http://dx.doi. org/10.4269/ajtmh.12-0698. 